\title{
Terahertz pulse imaging for tree-ring analysis: a preliminary study for dendrochronology applications
}

\author{
J B Jackson ${ }^{1,4,5}$, M Mourou ${ }^{1}$, J Labaune ${ }^{4}$, J F Whitaker $^{1}$, I N Duling III ${ }^{2}$, \\ S L Williamson ${ }^{2}$, C Lavier $^{3}, \mathrm{M} \mathrm{Menu}^{3}$ and G A Mourou ${ }^{4}$ \\ ${ }^{1}$ Center for Ultrafast Optical Science, University of Michigan, Ann Arbor, MI, 48109, USA \\ ${ }^{2}$ Picometrix, Advanced Photonics, Inc., Ann Arbor, MI, 48104, USA \\ ${ }^{3}$ Center for Research and Restoration of the Museums of France, Palais du Louvre, Paris, 75041, France \\ ${ }^{4}$ Institut Lumière Extrème, ENSTA—Ecole Polytechnique, Palaiseau, 91761, France \\ E-mail: j-bianca.jackson@ensta.fr
}

Received 9 December 2008, in final form 21 April 2009

Published 10 June 2009

Online at stacks.iop.org/MST/20/075502

\begin{abstract}
Time-domain terahertz reflection imaging is presented as a novel method of measuring otherwise inaccessible tree rings in wooden cultural heritage for the purpose of tree-ring crossdating. Axial and lateral two-dimensional terahertz images of obscured ring patterns are statistically validated with respect to their corresponding optical photographs via adapted dendrochronological methods. Results are compared to similar analysis of x-ray images of a wood specimen.
\end{abstract}

Keywords: terahertz, imaging, time-domain, reflectometry, tomography, wood, dendrochronology, cultural heritage

(Some figures in this article are in colour only in the electronic version)

\section{Introduction}

Since the development of terahertz sources and detectors over the last two decades, many spectroscopic and imaging applications have been pursued-most notably in homeland security, pharmaceutical and package monitoring and DNA analysis [1-4]. Recently, terahertz applications in the fields of cultural heritage and art conservation have gained interest, principally because terahertz radiation is non-contact, nondestructive and has the ability to penetrate non-metallic materials [5-8]. In the former area, terahertz-pulse reflection imaging has been used to study hidden sketch and paint layers under thick, optically opaque materials such as paint and plaster [9]. In this paper, we will discuss the feasibility of extending this application to the study of wood found in cultural heritage. We will compare terahertz and x-ray tree-ring imaging techniques by adapting tree-ring analysis

\footnotetext{
5 Author to whom any correspondence should be addressed.
}

methods used in the science of dating wooden objects, also known as dendrochronology.

Dendrochronology is the scientific method of using trees to create a time line for an object or place [10-12] and treering analysis is the first essential step of the process. Trees are an excellent tool for dating because-as a reflection of their environment - they are sensitive to both natural (i.e., weather) and man-made (i.e., pollution) events that affect tree growth. The volume and density of wood growth are not only dependent on the cyclic seasonal changes that produce earlywood and latewood in all trees, but also on localized climate, soil and water conditions. This results in the growth of tree rings unique to a specific period of time and place, so that patterns of wide and narrow ring growth accumulate over years during the lifetime of the tree. Therefore, trees growing in the same forest or biotope can be dated to each other (i.e., crossdating) by comparing similar ring patterns. Sequences of the unique ring patterns, or series, can be combined into a reference chronology for any particular location such that any tree-ring sample from that region can be compared 
to the reference and dated. Climate patterns can progress both linearly and nonlinearly over extended periods of time; therefore, longer ring series can allow for a more precise chronology [13].

Many past and present tree-ring measurement techniques are decidedly invasive, with tedious manual endeavours that include cutting, sand abrasion, acid-corrosion and-more recently - the laser-sandblasting of wooden objects, being employed [14]. Conservators desire to refrain from using these techniques, as they threaten the integrity of the works and erase traces of craftsmanship - both of which are of great interest to art conservators and historians. There are a few mechanically non-destructive techniques which can provide satisfactory measurement of tree rings in wooden objects [15], including ultrasonic [16,17], nuclear magnetic resonance (NMR) [18], neutron [19] and ion radiation imaging [20,21]. Currently, Okochi et al have produced exceptional results by resolving tree rings as narrow as $0.15 \mathrm{~mm}$ using soft $\mathrm{x}$-ray radiography (XRR), in addition to resolving rings in threedimensional painted wooden folk art using micro-focus $\mathrm{X}$-ray computed tomography (XRCT) [22].

There are portable terahertz systems which allow measurements to be performed in diverse environmental settings [23] comparable to that of portable ultrasonic, NMR [24] and X-ray scanners [25]. However, terahertz imaging has the versatility of being performed in either the transmission or reflection geometry. Terahertz transmission imaging has previously been proposed for the densitometric measurement of wood for tree-ring dating [26], as it is safer and less destructive than the several aforementioned methods [27]. An advantage of pulsed-terahertz reflectometry is that the semitransparency of most dielectric materials and the changes in reflectivity of the pulse-due to refractive index or optical density differences - can be exploited in order to image the spatial characteristics of materials buried beneath visibly opaque surface layers. The short-time-duration nature of terahertz pulses allows one to accurately isolate and distinguish depth information from different interfaces within an object, while the wide bandwidth of the terahertz pulses aids in spectroscopically discriminating buried materials.

We have investigated and implemented two methods of reflection-mode time-domain terahertz pulse imaging for resolving tree-ring information to facilitate the tree-ring dating of artefacts. We sampled rings in the tangential (parallel to ring growth) and cross-sectional (parallel to grain fibres) planes, as well as through the layers of primer and paint. We have comparatively analysed the tree-ring measurements of the terahertz images of the wood specimens with their respective photographs in order to determine the statistical accuracy of the images. Lastly, we compare the quality of our results to similar measurements of wood performed using x-ray imaging methods.

\section{Methodology}

The reflection measurements were carried out using a Picometrix QA-1000 terahertz time-domain imaging system [28], consisting of a femtosecond laser fibre-coupled to

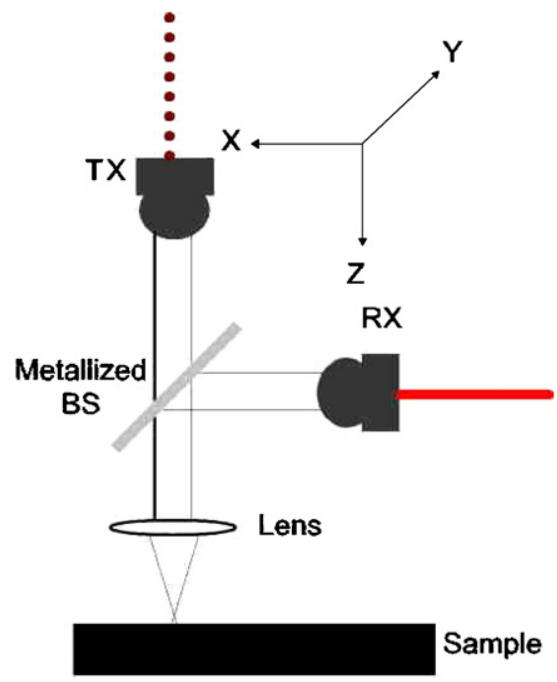

Figure 1. Diagram of co-linear terahertz generation and detection scheme.

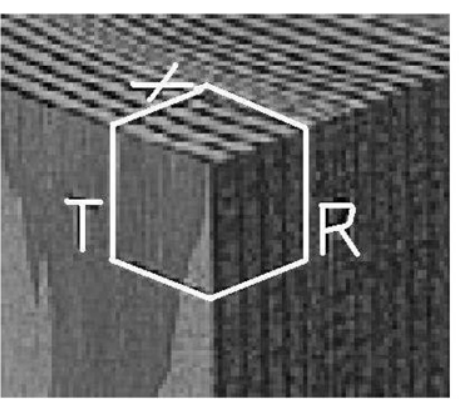

(a)

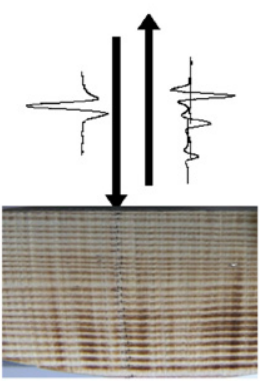

(b)
Figure 2. (a) The principal planes of wood are cross-sectional (X), radial (R) and tangential (T) and (b) a diagram of 'axial scan' probing of a wood specimen in the tangential plane.

an XYZ-translatable, photoconductive and co-linear $\mathrm{THz}$ transceiver. The emitter and hyper-hemispheric silicon lens combination produces a slowly expanding, free-space terahertz beam, which partially transmits through a metalized pellicle and is focused with an aspheric lens $(\mathrm{NA}=1)$ onto the sample plane at normal incidence. The reflected $\mathrm{THz}$ beam is collected by the same lens and partially reflected onto the photoconductive receiver, situated at $90^{\circ}$ with respect to the THz emitter (figure 1). One hundred waveforms were acquired per second, with 4 to 18 waveforms moving-averaged into a single pixel depending on sample area size.

The rings of the wood specimen were sampled using both $\mathrm{THz}$ ranging and en face tomographic methodologies, which we will call here 'axial scans' and 'lateral scans', respectively. An 'axial scan' image is a time-domain-based cross-sectional image of the region-of-interest of a specimen (figure 2(b)). In this case, the rings of the wood specimen were measured with the $\mathrm{THz}$ beam probing the wood in the tangential plane (figure 2(a)) with the focus of the beam positioned such that the depth of the focus $\left(2 z_{\mathrm{o}} \sim 1 \mathrm{~cm} @ 0.2 \mathrm{THz}\right)$ was entirely within the sample. The vertical dimension of an axial scan image corresponds to the signal amplitude in time, while the 


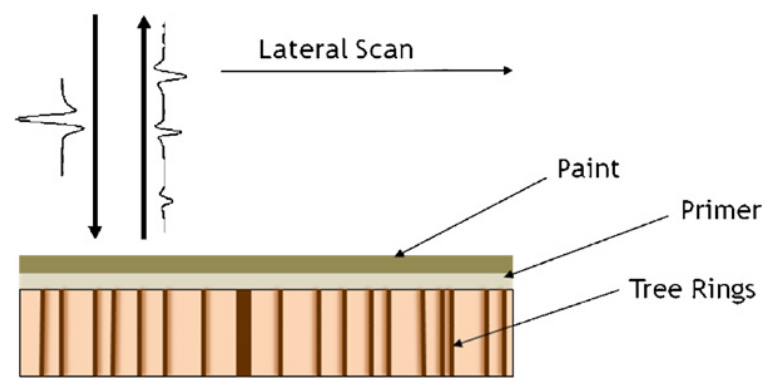

Figure 3. Diagram of the 'lateral scan' probe of a wood specimen.

horizontal dimension corresponds to the amplitude of the timedomain signal along the $x$-position.

For the en face tomographic measurements (figure 3), the $\mathrm{THz}$ beam probed the wood normal to either the longitudinal or the end-grain surface. Since there is a difference in optical density for the light (earlywood) and the dark (latewood) rings, the information on the wood surface obscured by paint can be extracted by analysing only the terahertz pulse reflected from the coating/wood interface.

In addition to varnishing wood for frames, furnishings, aesthetic room structures, etc, it was standard for artists to create paintings on thin wood panels until the ultimate ubiquity of canvas by 17 th to 18 th centuries [29]. Hence, there is interest in determining the ring patterns that exist under the paint within these framed substrates without removing the frame or otherwise disturbing the integrity of the painting. As a result, four sample wood specimens were studied to simulate these multilayer systems:

- an uncoated, seasoned pine (pinus sp.) dowel, plain-sawn cut on top for a flat surface;

- a seasoned pine (pinus sp.) plain-sawn newel post cap, uncoated (outer face) and coated (inner face) with three layers of acrylic varnish;

- a seasoned walnut (juglan sp.) tree stem cross-section, uncoated and coated with a layer of primer (calcium sulfate gesso and animal skin glue);

- a seasoned walnut (juglan sp.) tree stem cross-section from the same region, coated with layer of primer and a layer of commercially prepared lead white oil paint.

Pine was selected because it was an easily available conifercommonly used in North American carpentry-and has very well defined earlywood and latewood zones. It is immensely valuable for dendrochronology, with crossdating possible across subspecies and regions and chronology records available in international databases. Walnut is another popular wood for carpentry, often prized for its colours and texture. It is semi-diffuse porous, with gradually varying pore size and zone definition. Fewer dendrochronological records exist for walnut trees because they tend to favour stable ecological environments and grow in very small clusters; however, hetero-synchronized crossdating may be possible if the source location of the wood is known and oak chronologies from the same biotope are available.

Dendrochronologists commonly use software-such as COFECHA, Personal Analysis System for Tree-Ring
Research (PAST) or WinDENDRO_to aid this measurement, analysis and crossdating of ring-series patterns. The programs CooRecorder 7 and CDendro 7 by Cybis ${ }^{\mathrm{TM}}$ [30] were selected for the purpose of comparing photographs of the wood samples to their terahertz images, because of their low cost and userfriendly simplicity. CooRecorder 7 allows the user to import an image of the wood sample, identify the ring coordinates and then export the normalized ring width series to be compared to another sample or a standardized reference from an established database. Tree-ring series are crossdated both graphically and statistically for precision. The crossdating tool, CDendro 7 , enables the user to perform a ring-width transformation, which detrends the series for individual tree growth rates. For woods such as oak, pine or walnut, the Hollstein transformation was recommended:

$$
y(\text { Hollstein })_{i}=\log \left(\frac{y_{i}}{y_{i+1}}\right),
$$

where $y_{i}$ and $y_{i+1}$ are sequential ring widths. The ring series can then be graphically represented and offset to visually match the reference chronology.

For accuracy, the graphical-chronology match should be statistically verified. The three most common statistical parameters used for crossdating are Gleichlaufigkeit (GLK), correlation coefficient $\left(c_{\text {coeff }}\right)$ and an adapted student $t$-test [31]. The GLK represents the percentage slope equivalence of a given sample and reference with the overlapping parts of the two series. The correlation coefficient is a percentage of overall similarity between the two series. It is given by

$$
c_{\text {coeff }}=\frac{\sum_{i}\left(s_{i}-\bar{s}\right)\left(r_{i}-\bar{r}\right)}{\sqrt{\sum_{i}\left(s_{i}-\bar{s}\right)^{2}\left(r_{i}-\bar{r}\right)^{2}}},
$$

where $r_{i}$ and $s_{i}$ are the respective Hollstein transformed ring values of the reference and sample, and $\bar{r}$ and $\bar{s}$ are the mean values of the transformed ring series. A perfect match would correspond to $c_{\text {coeff }}=1.0$, while a perfect mismatch is $c_{\text {coeff }}=$ 0.0 . The correlation coefficient becomes more valuable when taking into account the length $(N)$ of overlapping series and therefore a $t$-test is used where

$$
t \text {-test }=\frac{c_{\text {coeff }} \sqrt{N-2}}{\sqrt{1-c_{\text {coeff }}^{2}}} .
$$

The ring series were measured along several radiistarting from the outside of the oldest latewood ring and continuing towards the pith-for each wood specimen's photographic reference and terahertz image. The ring series for each photograph were averaged to produce a single reference chronology (table 1). For the terahertz images, not all of the rings were resolvable along a particular radius; therefore, it was necessary to take ring blocks from different radii and overlap them to produce longer series. The ring series went through an alignment procedure where (1) they were graphically matched to each other and optimized using the GLK and $c_{\text {coeff }},(2)$ they were averaged to produce a mean sample chronology and (3) the mean sample chronology was compared to the reference chronology. Since the pine 2 sample was tangentially—not radially_cut, the ring widths were not 
Table 1. Average statistical parameters of photographic reference time series with respect to the reference chronology (OF/UC is the uncoated outer face and IF/C is the coated inner face of the specimen).

\begin{tabular}{lllllll}
\hline Sample & GLK & Overlap & $c_{\text {coeff }}$ & $c_{\text {coeff }}$ threshold & $t$-test & $t$-test threshold \\
\hline Pine 1 & $0.79 \pm 0.13$ & $19 \pm 5$ & $0.83 \pm 0.06$ & 0.77 & $6.1 \pm 1.2$ & 4.9 \\
Pine 2 (OF/UC) & $0.89 \pm 0.06$ & $24 \pm 8$ & $0.89 \pm 0.05$ & 0.84 & $9.2 \pm 1.5$ & 7.7 \\
Pine 2 (IF/C) & $0.84 \pm 0.07$ & $33 \pm 10$ & $0.82 \pm 0.09$ & 0.71 & $8.6 \pm 2.6$ & 6.0 \\
Walnut & $0.82 \pm 0.11$ & $14 \pm 3$ & $0.84 \pm 0.11$ & 0.73 & $6.1 \pm 2.5$ & 3.6 \\
\hline
\end{tabular}

equivalent for the two surfaces. This is a common problem for dendrochronologists with plain-sawn wood; hence it is important to obtain as much information to characterize the wood as possible.

The disadvantage of developing a novel measurement application is evident in the lack of an established rubric with which to precisely judge the statistical quality of our results. Acceptable GLK and $t$-test scores can vary depending on wood type and the ring-series length. Our assessment guidelines were influenced by Haneca et al [32] and Sass-Klassen et al [33] and their respective work in establishing new reference chronologies for the dendroprovenancing of timber. In the Sass-Klassen paper, the GLK must be at least 0.5 to be of any statistical significance in the ring series matches. For pine wood with a minimum of 40 rings, the $t$-test scores above 6.0 were definite matches and the scores below 3.0 most likely were not. In the Haneca paper, examples were given for the $t$-test scores ranging between 5.0 and 7.5, resulting in an acceptable $t$-test threshold of $6.0 \pm 25 \%$ for matching treering series to a reference chronology. In this case, the time series are longer with lower correlation coefficients. For our reference chronologies, the overlapping records are shorter but the correlation is high; therefore, both the $t$-test and $c_{\text {coeff }}$ values will be considered most relevant for judging good matches. The ranges of acceptability for the median values of the specimen's crossdating statistics will then be determined by the $1 /$ e standard deviation errors of the reference statistics.

\section{Results and discussion}

Figure 4 shows the time-domain reflection signature of the terahertz probe pulses incident on the side of the dowel normal to the tree rings seen in figure $2(b)$. The first reflection is from the air/pulp interface. The subsequent reflections occur at the well-defined boundaries between the earlywood and latewood growths, where there is a significant change in wood density resulting in Fresnel reflections. The refractive indices of the earlywood and latewood are approximately 1.12 and 1.251.38 , respectively. Precise calculation of the latewood index is not feasible under these circumstances due to the quasigradient composite structure of wood and the dependence of the propagation path length on the fibre orientation.

Due to the short focal depth of the THz beam with respect to the specimen thickness and attenuation and the scattering loss, only a limited number of rings produced reflections of significant amplitude. The bright lines of the axial scan image in figure 5 represent the strongly reflected $\mathrm{THz}$ signal at the earlywood/latewood boundaries, and thus the distance between the white lines are comparable to ring widths. The

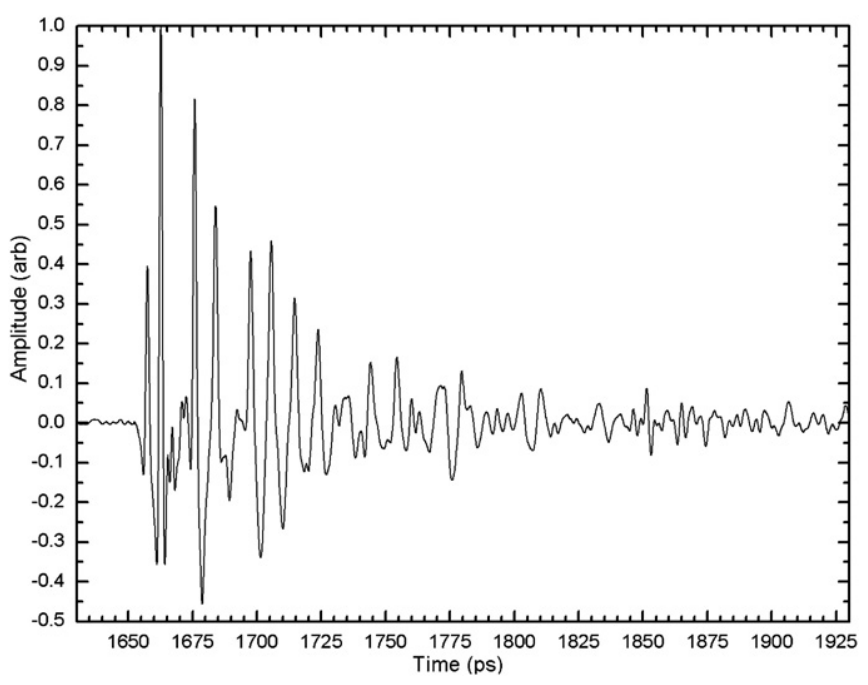

Figure 4. Time-domain waveform of the terahertz signal reflected from the pine 1 wood sample.

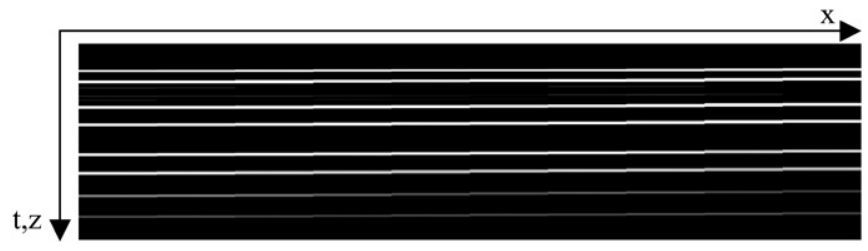

Figure 5. Terahertz axial scan image of the signal reflected from the pine 1 wood specimen (dimensions: $20 \mathrm{~mm} \times 320$ ps with $2 \mathrm{~mm} \times$ 0.0718 ps pixel size).

Table 2. Statistical parameters of pine 1 axial scan with respect to its reference chronology.

\begin{tabular}{lll}
\hline Parameter & Average of all ring series & Mean ring series \\
\hline$c_{\text {coeff }}$ & $0.84 \pm 0.02$ & 0.84 \\
$t$-test & $3.1 \pm 0.20$ & 3.1 \\
Overlap & $6 \pm 0$ & 6 \\
\hline
\end{tabular}

image colour threshold was limited, such that only signals greater than $12.5 \%$ of the largest signal were included. As the reflection amplitudes decrease, the lines become greyer. The amplitudes of secondary Fresnel reflections or echoes were determined to be insignificant $(0.01-0.3 \%)$ in comparison to primary reflections. However, system noise and diffuse scattering from the gradient latewood/earlywood boundary were a concern. Nonetheless, the distribution of the reflections remains consistent with the tree-ring distribution.

Table 2 shows the results of comparing the pine 1 specimen's collection of axial-scan ring widths with its reference chronology. The ring distribution was highly uniform within the scanned area; therefore, there was 


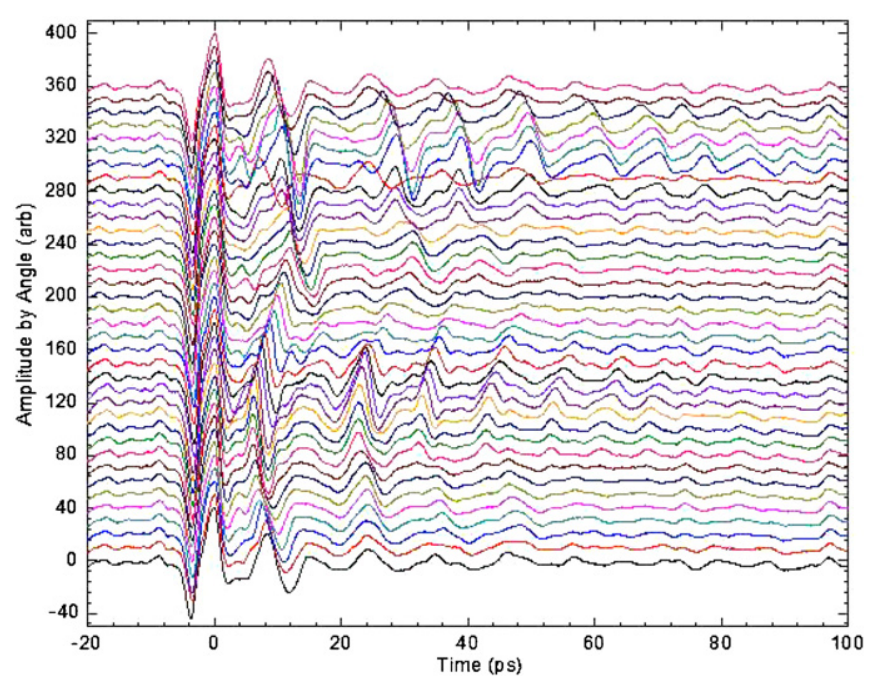

Figure 6. Time-domain amplitude waveforms of pine specimen over $360^{\circ}$ rotation.

no significant difference between the ring series for each measurement. The correlation coefficients for the axial scan image were greater than the threshold value for the pine specimen. Unfortunately, very few rings were resolved using this technique; thus, the $t$-test scores were significantly below the threshold value.

There are, however, limitations to this method of measuring tree rings. If the pulse separation is sufficient, information about each respective surface may be resolved independently. Using Buxton's criterion [34], the axial resolution of a depth measurement is maximized if the distance between the central maxima of the main lobes of the adjacent pulses of interest is greater than or equal to the full width at half maximum (FWHM) of the narrowest pulse. In our case, with a FWHM of approximately $0.8 \mathrm{ps}$, the narrowest ring separation we would be able to resolve for pine would be $100 \mu \mathrm{m}$. As such, this measurement scheme would benefit from a narrow temporal pulse.

Additionally, Reid et al [35] studied the terahertz birefringence and attenuation properties of wood in a transmission geometry. They were able to demonstrate that diattenuation and a frequency-independent time delay of the pulse occur as it propagates through the wood and is attributable to the scattering of the radiation by the non-random distribution of spindly-shaped longitudinal cells called fibrils. Therefore, in order to further reduce signal attenuation due to scattering and Fresnel losses, it was necessary to optimize the fibre alignment with the polarization of the terahertz beam. In figure 6 , the separation of the reflections from the first and second interfaces varies cyclically. The reflections from the subsequent layers are enhanced or attenuated as the fibres of the wood specimen are rotated from $0^{\circ}$ through $360^{\circ}$ in the incidence plane and with respect to the linear polarization of the terahertz pulse. Thus, the orientation should be optimized for maximum interface reflections, with a minimum time delay between them. This technique for determining fibre orientation may be valuable for providing insight into the reasons that a carpenter or artist selects their cuts of wood or how that wood has warped over time.

Figures $8(a)$ and $(b)$ show the laterally scanned images of the unvarnished (top) and varnished (bottom) pine 2 specimen sections seen in figure 7 . In this case, the primary pulse reflection is from the air/wood interface for the unvarnished specimen, or the superimposed pulse reflections from the air/varnish and varnish/wood interfaces for the varnished specimen. The images were calculated by integrating the power spectra of the reflection from the specimen from 0.5 to $0.8 \mathrm{THz}$ and 0.5 to $1.0 \mathrm{THz}$, respectively, to optimize the spatial resolution $\left(d_{\min } \sim 250 \mu \mathrm{m}\right.$, according to the Rayleigh criterion for the maximum frequency) and signal to noise. For finer tree-ring distributions, it may be recommended to integrate the signal at narrower bandwidth and/or higher frequencies. For low signal to noise, this would be better as a supplemental, rather than a principal, image for measurement because of the increased risk of missing or falsely measuring rings.

Using the CooRecorder software, the tree-ring series were repeatedly measured from the left and right sides of the centre pith and aligned with each other by optimizing the GLK and overlap. This increased the number of tree-ring series measured from a single specimen and demonstrated the radial scaling of tree-ring growth. Therefore, the quality of the graphical matches for individual series and their block GLK and $c_{\text {coeff }}$ analyses suggests that combining short blocks into

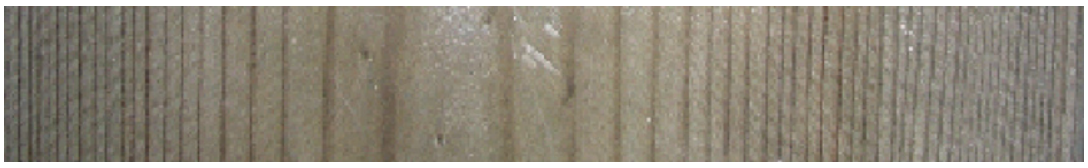

Figure 7. Optical photograph of a segment of the inner face of the pine 2 wood specimen.

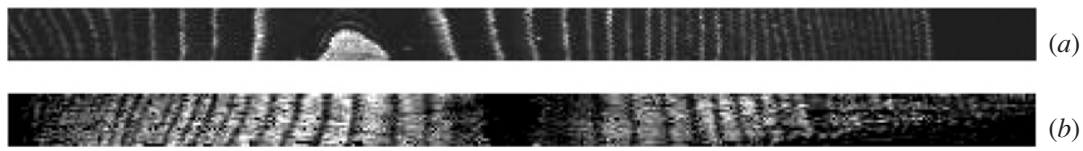

Figure 8. THz images of pine 2 wood specimen $(a)$ unvarnished and $(b)$ varnished $(100 \mathrm{~mm} \times 10 \mathrm{~mm}$ dimensions, $0.25 \mathrm{~mm} \times 1 \mathrm{~mm}$ pixel size). 


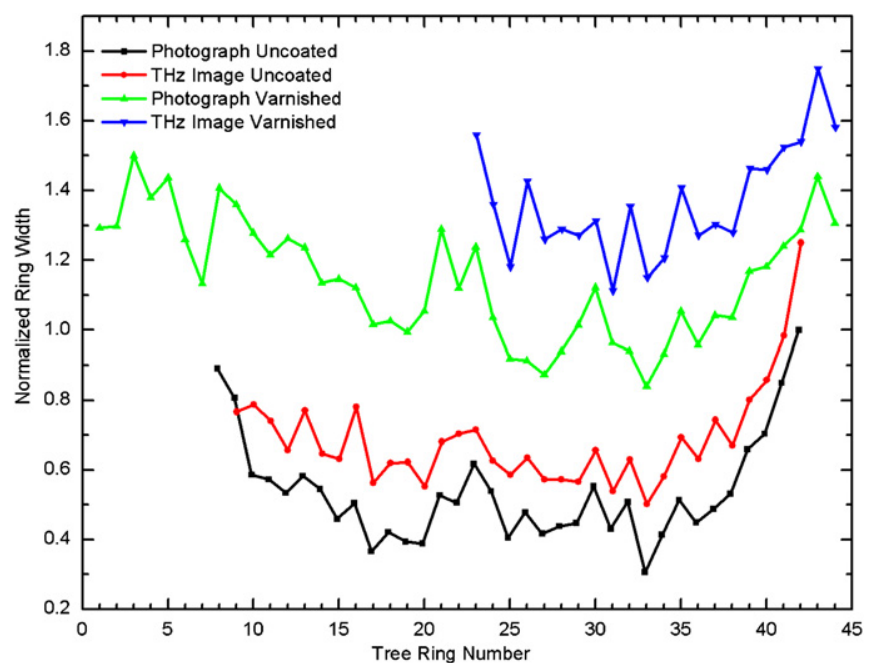

Figure 9. Comparison of mean tree-ring series of a photograph and a terahertz image of the pine 2 wood specimen.

longer tree-ring series may actually provide higher $t$-test results and more precise crossdating matches.

Figure 9 compares the arithmetic means of the tree-ring series collections for the unvarnished and varnished specimen to their unvarnished and varnished reference chronologies. Here, the rings are measured from the outermost ring and the 35th ring corresponds to the last before the pith. The unvarnished image produces a more precise overall tree-ring series replication, since the more tightly spaced tree rings were easier to resolve. However, for the THz image of the varnished specimen, several individual ring-width blocks within the series had strong correlation to both the photographic reference and the terahertz image of the unvarnished sample. For the unvarnished pine 2 specimen in table 3 , the $c_{\text {coeff }}$ and $t$-test values of the mean ring series were $2.4 \%$ and $22 \%$ above the threshold values of the photographic reference. For the varnished specimen (table 4), the correlation coefficient was $4 \%$ above the threshold for the photographic reference of the same face. Unfortunately, creating an extended mean ring series did not improve the $t$-test scores, resulting in
Table 3. Statistical parameters of the $0.5-0.8 \mathrm{THz}$ power integration image of the unvarnished pine 2 specimen with respect to pine 2 outer face reference chronology.

\begin{tabular}{lll}
\hline Parameter & Average of all ring series & Mean ring series \\
\hline$c_{\text {coeff }}$ & $0.75 \pm 0.23$ & 0.86 \\
$t$-test & $6.7 \pm 3.7$ & 9.4 \\
Overlap & $23 \pm 12$ & 33 \\
\hline
\end{tabular}

Table 4. Statistical parameters of $0.65-1.05 \mathrm{THz}$ power integration of the varnished pine 2 wood specimen with respect to pine 2 inner face reference chronology.

\begin{tabular}{lll}
\hline Parameter & Average of all ring series & Mean ring series \\
\hline$c_{\text {coeff }}$ & $0.76 \pm 0.18$ & 0.74 \\
$t$-test & $4.3 \pm 3.1$ & 4.8 \\
Overlap & $10 \pm 5$ & 21 \\
\hline
\end{tabular}

an unsatisfactory time series matching at $25 \%$ below the threshold.

The en face terahertz image of the uncoated walnut 1 specimen was compared to the optical photograph of the uncoated walnut 1 specimen, shown in figure 10. The terahertz image was obtained by calculating the second-order moment of the first reflected time-domain pulse (from the surface of the specimen) between 0.4 and $0.7 \mathrm{THz}$ :

$$
M_{\gamma}=\int_{v_{i}}^{v_{f}} S(\nu) v^{\gamma} \mathrm{d} \nu .
$$

The moment order is $\gamma, S(v)$ is the power spectral density and $v_{i}$ and $v_{f}$ are the boundaries of the frequency range of interest. When $\gamma$ is equal to zero, the equation is equivalent to the power spectra integration used to calculate figure 8. Different data reduction methods are sometimes required. Thus, in this case, the contributions of higher spectral frequencies optimize the spatial contrast between the reflectivities of earlywood and latewood.

Visually, most of the tree rings were resolvable for the uncoated walnut specimen. However, statistically the individual series (table 5) were not comparable to their

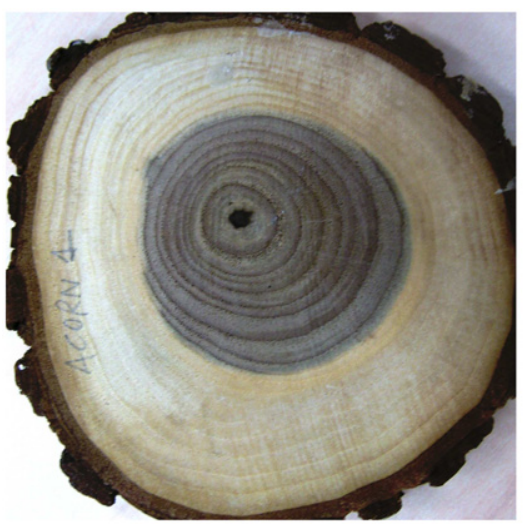

(a)

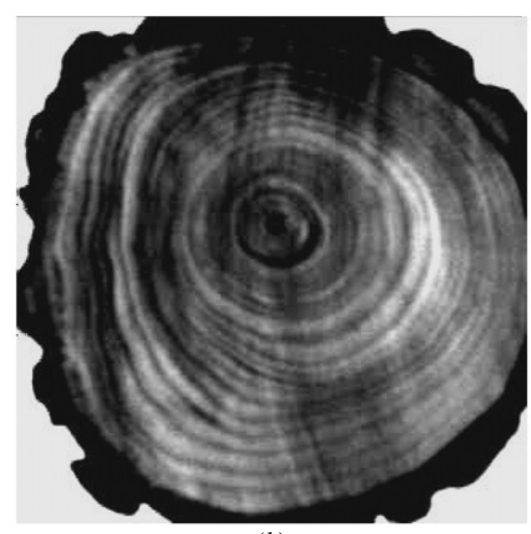

(b)

Figure 10.(a) Optical photograph and $(b)$ terahertz image of the uncoated walnut sample. 


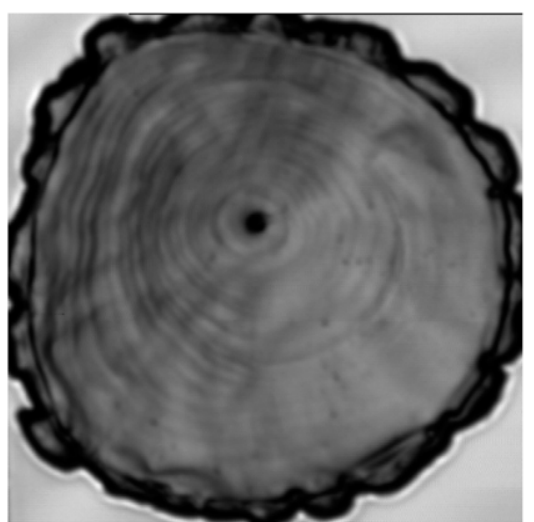

(a)

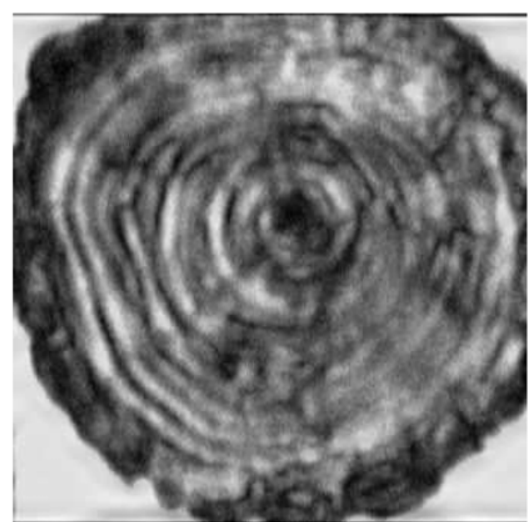

(b)

Figure 11. THz images of $(a)$ the walnut 1 specimen beneath the primer and $(b)$ the walnut 2 specimen beneath the primer and paint.

Table 5. Statistical parameters of the $\mathrm{M}_{2} \mathrm{THz}$ image of the uncoated walnut 1 specimen with respect to walnut reference chronology.

\begin{tabular}{lll}
\hline Parameter & Average of all ring series & Mean ring series \\
\hline$c_{\text {coeff }}$ & $0.67 \pm 0.10$ & 0.82 \\
$t$-test & $2.4 \pm 0.6$ & 5.2 \\
Overlap & $9 \pm 3$ & 15 \\
\hline
\end{tabular}

Table 6. Statistical parameters of the $\mathrm{M}_{2} \mathrm{THz}$ image of the primed walnut 1 specimen with respect to walnut reference chronology.

\begin{tabular}{lll}
\hline Parameter & Average of all ring series & Mean ring series \\
\hline$c_{\text {coeff }}$ & $0.70 \pm 0.22$ & 0.81 \\
$t$-test & $2.7 \pm 2.0$ & 5.0 \\
Overlap & $6 \pm 2$ & 15 \\
\hline
\end{tabular}

reference chronology, whereas the pine 2 specimen was comparable to its reference chronology, and the average $c_{\text {coeff }}$ was significantly below threshold. By combining all the series together into a mean series, the $c_{\text {coeff }}$ and the $t$-test were increased to $12.5 \%$ and $44.4 \%$ above threshold, respectively. This could be attributed to the subtle variations in the densities of earlywood and latewood widths from year to year being more significant for semi-diffuse porous wood than nonporous wood, thus slightly reducing the contrast. It should also be noted that there is no significant difference in reflectivity between the heartwood (dark centre) and the sapwood (tan outer) regions in this terahertz range.

For the primed specimen (figure 11(a)), fewer sections of the specimen were resolvable; hence, the measured individual tree-ring series were shorter than those for the uncoated specimen. Nonetheless, there were overlapping segments which enabled continuous full length mean series that fit nearly as well to the reference chronology as the uncoated specimen (table 6). The correlation coefficient and the $t$-test scores were $11 \%$ and $39 \%$ above threshold, respectively.

The terahertz image of the primed and painted walnut 2 specimen (figure 11(b)) was obtained by integrating the entire spectrum of the terahertz pulse reflected from the back surface of the specimen (i.e., the shadow). For this particular specimen, the pulse reflections from the air/paint,
Table 7. Statistical parameters of the THz image of the primed and painted walnut 2 specimen with respect to walnut reference chronology.

\begin{tabular}{lll}
\hline Parameter & Average of all ring series & Mean ring series \\
\hline$c_{\text {coeff }}$ & $0.53 \pm 0.15$ & 0.73 \\
$t$-test & $1.8 \pm 0.8$ & 3.9 \\
Overlap & $9 \pm 2$ & 15 \\
\hline
\end{tabular}

paint/primer and primer/wood interfaces were too closely overlapped for adequate temporal resolution, thus reducing the contrast between the early and late wood. As the terahertz pulse propagated through the specimen, however, the contrast strength between the two wood densities improved the ability to resolve the rings. The measured tree-ring series were then compared to the original walnut 1 reference chronology. Ultimately, a direct ring measurement would be preferred to this indirect technique, although adequate statistical results (table 7) were obtained when matching the mean ring series of two different walnut specimens from the same region. The $c_{\text {coeff }}$ was at threshold and the $t$-test scores were just $8.3 \%$ above the threshold value. Future work will take advantage of reflection cancellation techniques, such as those mentioned in [36], which may improve the resolution of the temporally overlapped primer and paint layers.

\section{Additional discussion}

In order to validate our measurement and analysis techniques, we decided to apply the same analysis to a photograph and two x-ray measurements, performed by Okochi et al, on a cross-sectional cut of diffuse-porous Japanese beech wood (Fagus crenata Blume) [22]. It is a popular wood for Japanese folk craft and a master chronology network is in progress. Soft XRR and XRCT are widely used in wood densitometry. However, recent advances have been made to improve the spatial resolution of XRCT measurements-which also has the advantage of digital image processing over film for XRR. XRR and XRCT have pixel resolutions of $21 \mu \mathrm{m}$ and $24 \mu \mathrm{m}$ with $400 \mu \mathrm{m}$ and $7 \mu \mathrm{m}$ focal spot sizes respectively. That is 


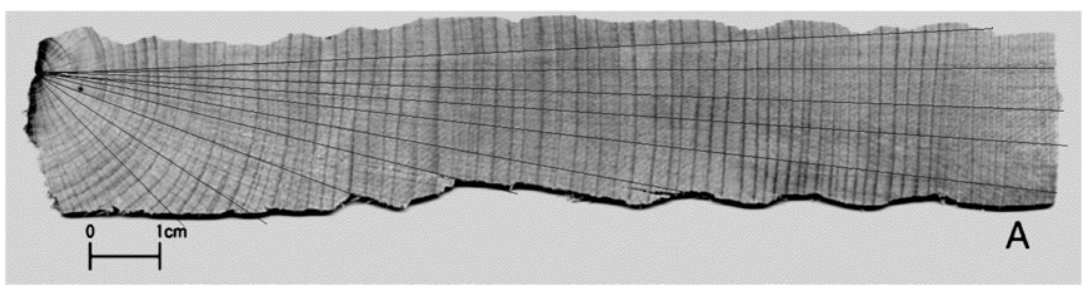

Figure 12. Photographic image of Japanese beech wood [22].

Table 8. Statistical parameters of the tree-ring series for the photograph of the Japanese beech specimen with respect to the mean reference chronology.

\begin{tabular}{lllllll}
\hline Sample & GLK & Overlap & $c_{\text {coeff }}$ & $c_{\text {coeff }}$ threshold & $t$-test & $t$-test threshold \\
\hline Beech & $0.81 \pm 0.08$ & $35 \pm 12$ & $0.77 \pm 0.16$ & 0.61 & $8.0 \pm 3.6$ & 4.4 \\
\hline
\end{tabular}

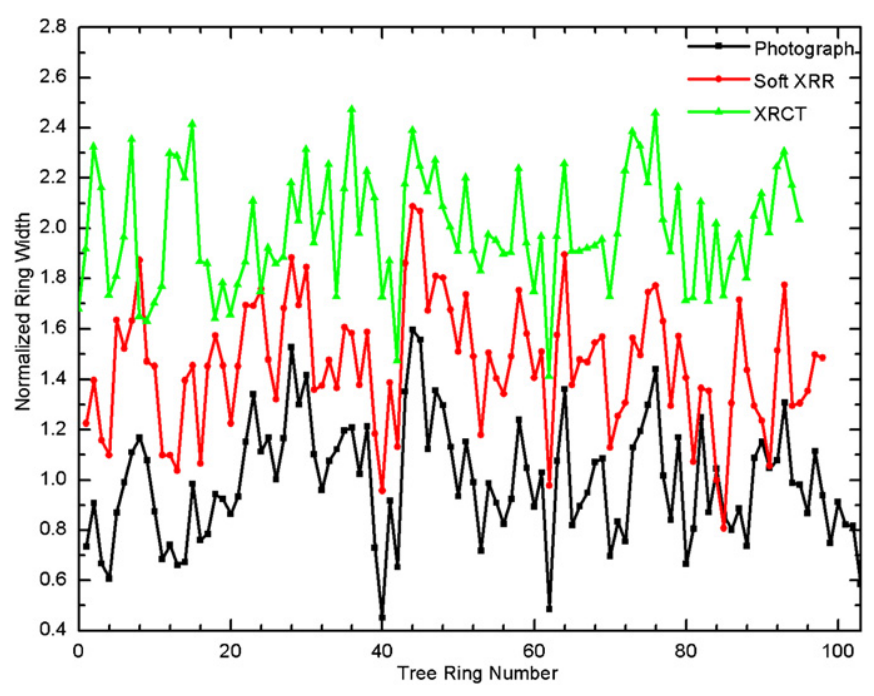

Figure 13. Comparison of normalized mean tree-ring series of the Japanese beech photograph, soft x-ray and x-ray CT images.

less than half the $10 \mu \mathrm{m}$ pixel resolution dendrochronologists generally demand for digital photography (figure 12).

For the photograph, the earlywood is lighter than the latewood in the heartwood region, while it appears darker in the sapwood region. Figure 13 shows a graphical comparison
Table 9. Statistical parameters of the soft-XRR image of the Japanese beech specimen [22].

\begin{tabular}{lll}
\hline Parameter & Average of all ring series & Mean ring series \\
\hline$c_{\text {coeff }}$ & $0.80 \pm 0.11$ & 0.81 \\
$t$-test & $8.2 \pm 3.7$ & 13.5 \\
Overlap & $33 \pm 12$ & 97 \\
\hline
\end{tabular}

Table 10. Statistical parameters of the micro-focus XRCT image of the Japanese beech specimen.

\begin{tabular}{llc}
\hline Parameter & Average of all ring series & Mean ring series \\
\hline$c_{\text {coeff }}$ & $0.63 \pm 0.14$ & 0.74 \\
$t$-test & $5.2 \pm 2.2$ & 11.1 \\
Overlap & $38 \pm 13$ & 103 \\
\hline
\end{tabular}

of the tree-ring series for each image. The x-ray images in figures 14 and 15 appear to resolve the tree rings more uniformly than the photograph-which may skew the results in table 8 . The $t$-test threshold are set artificially low because of the wide range in the overlapping segment lengths.

For the soft-XRR image, the individual ring series are comparable to that of the photograph, with respect to the reference chronology (table 9). The extended length of the mean ring series results in a $64 \%$ improvement on the

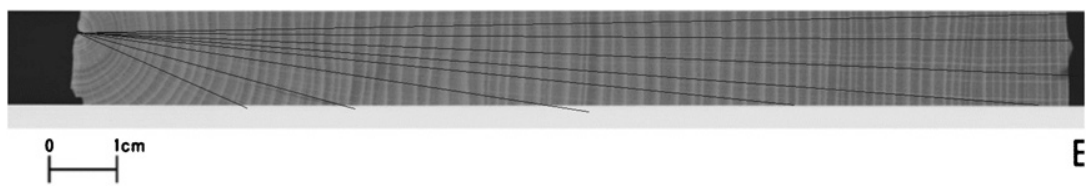

Figure 14. Soft-XRR image of the Japanese beech specimen [22].

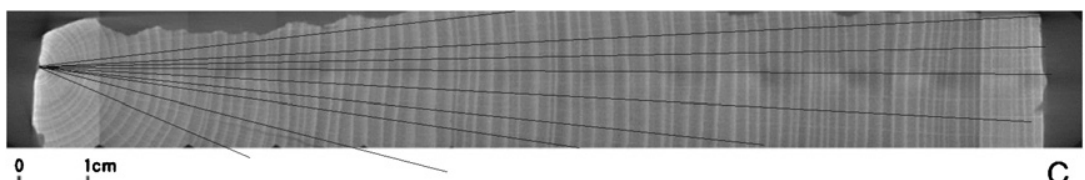

Figure 15. Micro-focus XRCT image of the Japanese beech specimen [22]. 
average $t$-test score, thus exceeding the threshold by $200 \%$. The precision of the X-ray CT image was good, but not as satisfactory as the soft-XRR image (table 10). The average $c_{\text {coeff }}$ and $t$-test scores of all the ring series were $3.4 \%$ and $18 \%$ greater than the threshold, respectively, while the mean series values were $21 \%$ and $150 \%$ greater.

In comparison to the terahertz image of the uncoated walnut specimen cross-section, the tree-ring boundaries of the beech specimen are very well defined. The overall sample length of the beech has an advantage over our walnut sample with respect to the $t$-test scores; nonetheless the correlation coefficients are comparable enough that terahertz imaging appears viable as an alternative measurement technique.

\section{Conclusions}

The ring-growth patterns of several wood specimens were investigated using terahertz time-domain reflectometry. Tree rings measured tangentially using ranging were not deeply penetrated, but had a satisfactory spatial correlation to their photographic reference. Tree rings imaged along the longitudinal and end-grain surfaces were spatially resolvable using en face tomography. The statistical accuracy of the ring series for the natural wood specimens was good in comparison with their reference chronologies; however, the ring series for the varnished specimen were unsatisfactorily matched. The tree rings for the primed and painted specimens were not uniformly resolved, but enough information was obtained from the terahertz measurements that their ring series could be satisfactorily compared to the reference chronology.

The THz-TDR measurements of wood presented in this paper were very preliminary. While more established imaging techniques, such as X-ray imaging, produced the most satisfactory images, there is still a promise that THz-TDR could be useful for the dendrochronological crossdating of wooden works of art, particularly panel paintings. Before tree-ring crossdating is possible, a thorough study should be performed to determine which wood pore structures (nonporous, ring-porous, ring-diffuse, etc) are suited for axial or lateral terahertz measurement in order to maximize the number of resolvable tree rings. The spatial resolution of measurements should also be improved by increasing the signal-to-noise at higher spectral frequencies. There are also many options for further exploration that include and go beyond crossdating applications. THz-TDR may be used to study stress and fatigue in wood as it ages or responds to external forces, by taking advantage of the inherent anisotropy of wood. It may also be used to assess water content or damage in artefacts and artwork.

\section{References}

[1] Mittleman D M, Jacobsen R H and Nuss M C 1996 T-ray imaging IEEE J. Sel. Top. Quant. Electron. 2 679-92

[2] Wang S and Zhang X C 2004 Pulsed terahertz tomography J. Phys. D: Appl. Phys. 37 R1-36

[3] Chan W L, Diebel J and Mittleman D M 2007 Imaging with terahertz radiation Rep. Prog. Phys. 70 1325-79
[4] Juuti M, Tuononen H, Prykäri T, Kontturi V, Kuosmanen M, Alarousu E, Ketolainen J, Myllylä and Peiponen K-E 2009 Optical and terahertz measurement techniques for flat-faced pharmaceutical tablets: a case study of gloss, surface roughness and bulk properties of starch acetate tablets Meas. Sci. Technol. 20015301

[5] Manceau J-M, Nevin A, Fotakis C and Tzortzakis S 2008 Terahertz time domain spectroscopy for the analysis of cultural heritage related materials Appl. Phys. B $90365-8$

[6] Köhler W, Panzer M, Klotzach U, Winner S, Helm M, Rutz F, Jördens C, Koch M and Leitner H 2006 Non-destructive investigation of paintings with THz-radiation European Conf. of Non-Destructive Testing 2006 Proc. Poster 181

[7] Fukunaga K, Ogawa Y, Hayashi S and Hosako I 2007 Terahertz spectroscopy for art conservation IEICE Electron. Express 4 258-63

Fukunaga K, Sekine N, Hosako I, Oda N, Yoneyama H and Sudoh T 2008 Real-time terahertz imaging for art conservation science J. Eur. Opt. Soc., Rap. Publ. 308027

[8] Oyama Y, Zhen L, Tanabe T and Kagaya M 2009 Sub-terahertz imaging of defects in building blocks $N D T \& E$ Int. 42 28-33

[9] Jackson J B, Mourou M, Whitaker J F, Duling I, Williamson S, Menu M and Mourou G 2008 Terahertz imaging for non-destructive evaluation of mural paintings Opt. Commun. 281 527-32

[10] Douglass A E 1937 Tree-ring work Tree-ring Bulletin 4 3-6 Douglass A E 1941 Crossdating in dendrochronology J. Forest. 39 825-31

[11] Nash S 2002 Archaeological tree-ring dating at the millennium J. Archaeol. Res. 10 243-76

[12] Fritts H C 1995 Dendrochronology Quaternary of the United States: Seventh Congress of the International Association of Quaternary Research pp 871-9

[13] Kuniholm P I 2001 The Handbook of Archeological Sciences (London: Wiley)

[14] Lesnino G 1994 The laser-sandblasting method: a new method for the qualitative annual ring analysis of conifers Wood Sci. Technol. 28 159-71

[15] Bucur V 2003 Techniques for high resolution imaging of wood structure: a review Meas. Sci. Technol. 14 R91-8

[16] Murray A, Mecklengurg M F, Fortunko C M and Green R E 1996 Air-coupled ultrasonic system: a new technology for detecting flaws in paintings on wooden panels J. Am. Inst. Conservat. 35 145-62

[17] Berndt H, Schniewind A P and Johnson G C 1999 High-resolution ultrasonic imaging of wood Wood Sci. Technol. 33 185-98

[18] Bucur V 2003 Nondestructive Characterization and Imaging of Wood (Heidelberg: Springer)

[19] Mannes D, Lehmann E, Cherubini P and Niemz P 2007 Neutron imaging versus standard $\mathrm{x}$-ray densitometry as a method to measure tree-ring density Trees 21 605-12

[20] Davis J and Wells P 1992 Computed tomography measurements on wood Ind. Metrol. 2 195-218

[21] Onoe M, Tsao J W, Yamada H, Nakumura H, Kogure J, Kawamura H and Yoshimatsu M 1983 Computed tomography for measuring annual rings of a live tree Proc. IEEE 71 907-8

[22] Okochi T, Hoshino Y, Fujii H and Misutani T 2007 Nondestructive tree-ring measurements for Japanese oak and Japanese beech using micro-focus x-ray computed tomography Dendrochronologia 24 155-64

[23] Jackson J B, Mourou M R, Whitaker J F, Duling I N, Menu M, Labaune J and Mourou G A 2008 Reflection pulse imaging of hidden Fresco paintings European Optical Society Annual Meeting TOM 2963 
[24] Rokitta M, Rommel E, Zimmermann U and Haase A 2000 Portable nuclear magnetic resonance imaging system Rev. Sci. Instrum. 71 4257-62

[25] Dubecký F, Perd'ochová A, Ščepko P, Zat'ko B, Sekerka V, Nečas V, Sekáčová M, Hudec M, Boháček P and Huran J 2005 Digital x-ray portable scanner based on monolithic semi-insulating GaAs detectors: general description and first 'quantum' images Nucl. Instrum. Methods Phys. Res. A $546118-24$

[26] Koch M, Hunsche S, Schuacher P, Nuss M C, Feldmann J and Fromm J 1998 THz-imaging: a new method for density mapping of wood Wood Sci. Technol. 32 421-7

[27] Walker G C, Berry E, Zinov'ev N N, Fitzgerald A J, Miles R E, Chamberlain J and Smith M A 2002 Terahertz imaging and international safety guidelines Proc. SPIE 4682 683-90

[28] Zimdars D 2005 High speed terahertz reflection imaging Proc. SPIE 5692 255-9

[29] Bauch J and Eckstein D 1970 Dendrochronological dating of oak panels of Dutch 17th c. paintings Stud. Conserv. 15 45-50
[30] http://www.cybis.se/forfun/dendro/index.htm

[31] Knibbe B 2004 Personal Analysis System for Tree-Ring Research 4-Instruction Manual (Vienna: SCIEM)

[32] Haneca K, Wazney T, Van Acker J and Beeckman H 2005 Provenancing Baltic timber from art historical objects: success and limitations J. Archeol. Sci. 32 261-71

[33] Sass-Klassen U, Vernimmen T and Baitinger C 2008 Dendrochronological dating and provenancing of timber used as foundation piles under historic buildings in The Netherlands Int. Biodeter. Biodegr. 61 96-105

[34] den Dekker A J and Van Den Bos A 1997 Resolution: a survey J. Opt. Soc. Am. A 14547

[35] Reid M and Fedosejevs R 2006 Terahertz birefringence and attenuation properties of wood and paper Appl. Opt. $452766-72$

Todoruk T M, Schneider J, Hartley I D and Reid M 2008 Birefringence of wood at terahertz frequencies Proc. SPIE $709970992 \mathrm{Q}$

[36] Hirsch O, Alexander P and Gladden L F 2008 Techniques for cancellation of interfering multiple reflections in terahertz time-domain measurements Microelectron. J. 39 841-8 